\title{
A note on maxima in random walks
}

\author{
Joseph Helfer \\ Department of Mathematics \\ Stanford Univ. \\ Stanford, CA 94305, U.S.A. \\ joj@stanford.edu
}

\author{
Daniel T. Wise* \\ Dept. of Math. \& Stats. \\ McGill Univ. \\ Montreal, QC, Canada H3A 0B9 \\ wise@math.mcgill.ca
}

Submitted: Jun 13, 2015; Accepted: Jan 13, 2016; Published: Jan 22, 2016

Mathematics Subject Classifications: 60G50, 05A19

\begin{abstract}
We give a combinatorial proof that a random walk attains a unique maximum with probability at least $1 / 2$. For closed random walks with uniform step size, we recover Dwass's count of the number of length $\ell$ walks attaining the maximum exactly $k$ times. We also show that the probability that there is both a unique maximum and a unique minimum is asymptotically equal to $\frac{1}{4}$ and that the probability that a Dyck word has a unique minimum is asymptotically $\frac{1}{2}$.
\end{abstract}

Keywords: Random walk, Dyck Words, Catalan Numbers

\section{Introduction}

A length $\ell$ walk is a sequence $w:\{1, \ldots, \ell\} \rightarrow\{ \pm 1\}$. The trajectory of $w$ is the sequence $\bar{w}:\{0, \ldots, \ell\} \rightarrow \mathbb{Z}$ defined by $\bar{w}(j)=\sum_{i=1}^{j} w(i)$. We define $\max (w)=\sup \{\bar{w}(j): 0 \leqslant$ $j \leqslant \ell\}$. The length $\ell$ walk $w$ is closed if $\bar{w}(\ell)=0$. Let $\mathcal{C}(n)$ denote the set of length $2 n$ closed walks and let $\mathcal{M}(n) \subset \mathcal{C}(n)$ denote the subset consisting of those walks $w$ for which there is a unique $i \in\{1, \ldots, 2 n\}$ such that $\bar{w}(i)=\max (w)$.

The paper centers around a combinatorial proof of the following theorem which was first proven by Dwass in [5]:

Theorem 2.4. $|\mathcal{M}(n)|=\frac{1}{2}|\mathcal{C}(n)|$ for each $n \geqslant 1$.

In [5], this is proven by a method that computes the probabilities of events in finite random walks by relating them to events in infinite random walks for which probabilities are more readily computed. This is a general analytic method used to compute a large

${ }^{*}$ Research supported by NSERC 
number of quantities including $\mathcal{M}(n)$ as well as the more general $\mathcal{M}(n, r)$ which we discuss in Section 3 .

As is often the case, a combinatorial proof offers other intuition and insight. In this case, we will see that our method generalizes to certain cases not amenable to Dwass's method.

In Section 2 we give a first proof of Dwass's result, which uses a method we will employ fundamentally in the text. A second more transparent proof is give in Section 6. This second proof uses that the number of Dyck words is the corresponding Catalan number. In Section 3 we recover Dwass's stronger result that there are precisely $\left(\begin{array}{c}2 n-r \\ r-1\end{array}\right)$ length $2 n$ closed walks attaining their maximum exactly $r$ times. In Section 4 we explain that the combinatorial proof generalizes to show that more general types of finite random walks have probability $\geqslant \frac{1}{2}$ of attaining a unique maximum. This conclusion does not assume that the walks are closed and allows an arbitrary distribution of step sizes. In Section 5 we show that the probability of having both a unique minimum and a unique maximum approaches $\frac{1}{4}$ as the length of a uniform closed walk increases. In Section 6 we show that the probability that a length $n$ Dyck word has a unique minimum approaches $\frac{1}{2}$ as $n \rightarrow \infty$.

\section{Dyck Words and Leads}

A Dyck word of length $2 n$ is a closed walk $w$ such that $\max (w)=0$. Let $\mathcal{D}(n)$ denote the set of length $2 n$ Dyck words. The number of Dyck words of a given length is the corresponding Catalan number:

Theorem 2.1. $|\mathcal{D}(n)|=\frac{1}{n+1}\left(\begin{array}{c}2 n \\ n\end{array}\right)$.

The lead of $w \in \mathcal{C}(k)$ is the number of values $i \in\{1, \ldots, 2 k\}$ with both $w(i)>0$ and $\bar{w}(i)>0$. For $0 \leqslant e \leqslant k$ let $\mathcal{L}(k, e) \subset \mathcal{C}(k)$ be the set of lead $e$ walks (so that $\mathcal{L}(k, 0)=\mathcal{D}(k))$. Thus $\mathcal{C}(k)=\sqcup_{e=0}^{k} \mathcal{L}(k, e)$.

Since $|\mathcal{C}(n)|=\left(\begin{array}{c}2 n \\ n\end{array}\right)$, Theorem 2.1 follows from the Chung-Feller Theorem which states that $|\mathcal{L}(k, e)|$ is independent of $e[2$. Among the many proofs of the Chung-Feller theorem is a bijective explanation given in [1, 6] the former of which traced the explanation to [4]. We now recount the bijection:

Lemma 2.2. For each $1 \leqslant e \leqslant k$ there is a bijection $\psi: \mathcal{L}(k, e-1) \rightarrow \mathcal{L}(k, e)$.

Proof. Let $w \in \mathcal{L}(k, e-1)$. Let $p>0$ be maximal such that $w(p)=-1$ and $\bar{w}(p)=-1$. Regard $w$ as a string in $\{ \pm 1\}$, and express $w$ as the concatenation $a x b$ where $a$ is the initial length $p$ subpath and $x$ is a single symbol (which is necessarily -1 ). Define $\psi(w)$ to be the sequence corresponding to $b x a$. This lies in $\mathcal{L}(k, e)$ since $a, x b, b x$ are all closed and the lead of $b x$ is one greater than that of $b$.

The map $\psi^{-1}$ is defined by recognizing the decomposition of $w \in \mathcal{L}(k, e)$ as a concatenation $b x a$ by declaring $b$ to be the length $n$ subword where $n$ is minimal such that $w(n+1)=-1$ and $\bar{w}(n+1)=0$. 


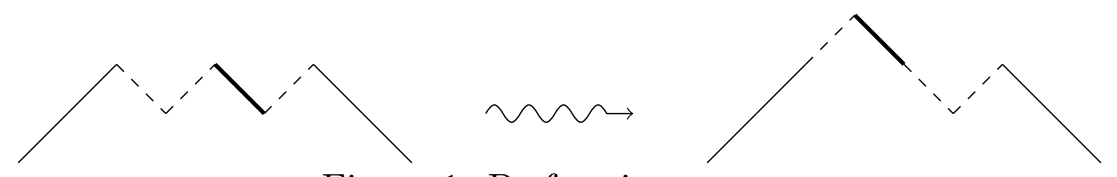

Figure 1: Performing a swap

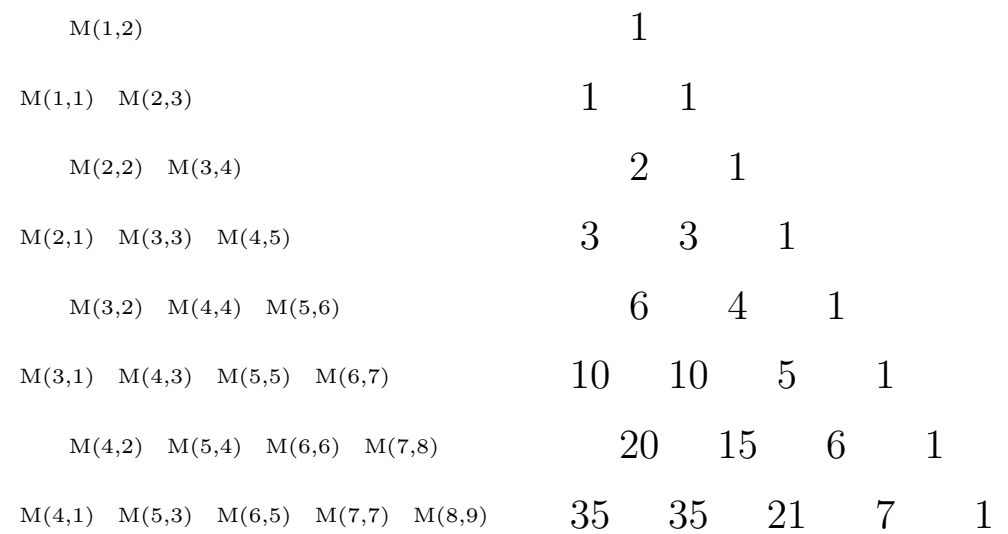

Figure 2: The base cases (1) are the entries $1,3,10,35, \ldots$ and the 1 at the top. The identity (2) states that an entry in Pascal's triangle is the sum of all the numbers in the diagonal path above it, e.g. $15=10+4+1$.

Remark 2.3. We utilize $\psi: \mathcal{L}(k, 0) \rightarrow \mathcal{L}(k, 1)$ whose crucial property is that $\psi(w) \in$ $\mathcal{M}(k)$ for $w \in \mathcal{L}(k, 0)$.

Theorem 2.4. $|\mathcal{M}(n)|=\frac{1}{2}|\mathcal{C}(n)|$ for each $n \geqslant 1$.

Proof. We describe a bijection $\Psi: \mathcal{C}(n)-\mathcal{M}(n) \rightarrow \mathcal{M}(n)$. Let $w \in \mathcal{C}(n)-\mathcal{M}(n)$. As $\operatorname{rank}(w) \geqslant 2$, we let $a$ be the nontrivial subsequence of $w$ with domain $\{p, \ldots, q\} \subset \mathbb{N}$ where $p-1, q$ are the minimal and maximal values of $\bar{w}^{-1}(\max (w))$. Note that $a \in$ $\mathcal{L}(q-p+1,0)$ and let $\psi(a) \in \mathcal{L}(q-p+1,1)$ be as provided by Lemma 2.2. Define $\Psi(w)$ to be the sequence obtained from $w$ by substituting $\psi(a)$ for $a$ as in Figure 1 . Note that $\Psi(w) \in \mathcal{M}(n)$ by Remark 2.3 .

An alternate proof is given in Section 6 .

\section{Counting walks of arbitrary rank}

The rank of a walk $w$ is $\left|\bar{w}^{-1}(\max (w))\right|$. For $r \geqslant 1$ let $\mathcal{M}(n, r) \subset \mathcal{C}(n)$ denote the subset of rank $r$ length $2 n$ closed walks. As $\mathcal{M}(n, 1)=\mathcal{M}(n)$, Theorem 2.4 states that $|\mathcal{M}(n, 1)|=\frac{1}{2}\left(\begin{array}{c}2 n \\ n\end{array}\right)=\left(\begin{array}{c}2 n-1 \\ n-1\end{array}\right)$. We now extend this result and count each $\mathcal{M}(n, r)$ :

Theorem 3.1. For $n, r \geqslant 1$ we have $|\mathcal{M}(n, r)|=\left(\begin{array}{c}2 n-r \\ n-1\end{array}\right)$.

Proof. Let $M(n, r)=\left(\begin{array}{c}2 n-r \\ n-1\end{array}\right)$. We first observe that the numbers $M(n, r)$ satisfy the following recursive definition (see Figure 2). 


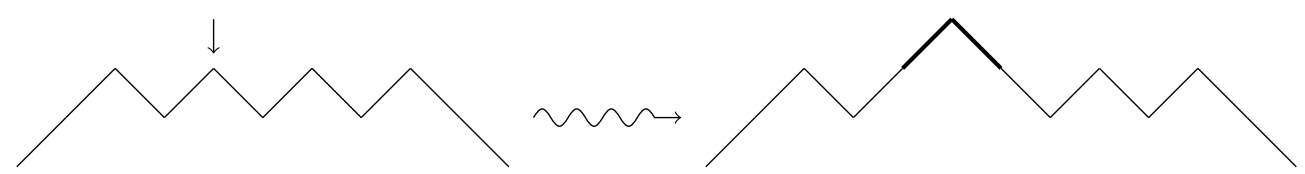

Figure 3: Inserting a peak at the third maximal peak from the right

The base cases are:

$$
M(n, 1)=\left(\begin{array}{c}
2 n-1 \\
n-1
\end{array}\right) \quad \text { and } \quad M(1,2)=1
$$

The inductive step for $n, r \geqslant 2$ follows by iterating Pascal's identity:

$$
M(n, r)=\sum_{j=r-1}^{n} M(n-1, j)
$$

We will show that Equations (1) and (2) are satisfied with $M(a, b)$ replaced by $|\mathcal{M}(a, b)|$, from which it follows that $|\mathcal{M}(n, r)|=M(n, r)$ for all $n, r \geqslant 1$ as desired.

Equation $\sqrt{1})$ is easy as Theorem 2.4 asserts that $|\mathcal{M}(n, 1)|=\left(\begin{array}{c}2 n-1 \\ n-1\end{array}\right)$ and obviously $|\mathcal{M}(1,2)|=1$.

To verify Equation (2), for each $n, r \geqslant 2$, we describe a bijection $\chi_{r}$ between $\bigcup_{j=r-1}^{n} \mathcal{M}(n-$ $1, j)$ and $\mathcal{M}(n, r)$. Let $w \in \bigcup_{j=r-1}^{n} \mathcal{M}(n-1, j)$. As $\operatorname{rank}(w) \geqslant r-1$, we may consider the $(r-1)$ th maximal peak of $w$, counted from the right. As in Figure 3 , we insert a peak at this point to obtain a path $\hat{w} \in \mathcal{M}(n)$. We then apply the map $\Psi^{-1}: \mathcal{M}(n) \rightarrow \mathcal{C}(n) \backslash \mathcal{M}(n)$ to $\hat{w}$ to obtain $\chi_{r}(w)$.

We must show that $\chi_{r}$ is injective and that its image is $\mathcal{M}(n, r)$. The former holds since a left-inverse to $\chi_{r}$ is obtained by first applying $\Psi$ and then removing the single maximal peak. The latter holds by Lemma 3.2 .

Lemma 3.2. Let $n, r \geqslant 2$, let $w \in \mathcal{M}(n, r)$ and let $w^{\prime}$ be obtained by removing the single maximal peak from $\Psi(w)$. Then $\operatorname{rank}\left(w^{\prime}\right) \geqslant r-1$.

Proof. The general case follows from the case where $w \in \mathcal{D}(m) \cap \mathcal{M}(m, r)$ so that $w^{\prime} \in$ $\mathcal{D}(m-1)$. We express $w$ as axb where the length $i$ of $a$ is maximal such that $i<m$ and $\bar{w}(i)=0$. Then $\Psi(w)=b x a$ by definition. $\operatorname{As} \operatorname{rank}(w)=r$, we have $\operatorname{rank}(a)=r-1$. Hence $\operatorname{rank}\left(w^{\prime}\right) \geqslant r-1$ as desired.

\section{Variable step lengths}

In this section we provide a different generalization of Theorem 2.4 we prove that for a random closed walk of variable step size, and with a nonzero fixed number of each type of step, the probability of attaining a unique maximum is at least $\frac{1}{2}$.

Definition 4.1. Let $S$ be a finite set. A length $\ell S$-walk $w$ is a sequence $\{1, \ldots, \ell\} \rightarrow S$. Let $\mathcal{W}_{S}(n)$ denote the set of length $n S$-walks. Let $v: S \rightarrow \mathbb{R}$. The $v$-trajectory of $w$ is 
$\bar{w}_{v}(j)=\sum_{i=1}^{j} v(w(i))$. The $v$-maximum of $w$ is $\max _{v}(w)=\max \left\{\bar{w}_{v}(j): 0 \leqslant j \leqslant \ell\right\}$. Let $\mathcal{M}_{v}(n) \subset \mathcal{W}_{S}(n)$ denote the subset consisting of those walks $w$ for which there is a unique $i \in\{1, \ldots, n\}$ such that $\bar{w}(i)=\max (w)$. The length $\ell S$-walk $w$ is $v$-closed if $\bar{w}_{v}(\ell)=0$. Let $\mathcal{D}_{v}(n) \subset \mathcal{W}_{S}(n)$ denote the set of length $N v$-closed $S$-walks with $v$-maximum 0 .

The proof of Lemma 2.2 can be carried over to this more general context:

Lemma 4.2. There is an injection $\psi_{v}: \mathcal{D}_{v}(n) \rightarrow \mathcal{M}_{v}(n)$

Proof. Let $w \in \mathcal{D}_{v}(n)$. Let $p<n$ be maximal such that $\bar{w}_{v}(p)=0$. Regard $w$ as a string in $S$, and express $w$ as the concatenation $a x b$ where $a$ is the initial length $p$ subpath and $x$ is a single symbol (which necessarily satisfies $v(x)<0$ ). Define $\psi_{v}(w)$ to be the sequence corresponding to $b x a$. This lies in $\mathcal{M}_{v}(n)$ since $a$ has $v$-maximum 0 and $\bar{b}_{v}$ obtains its unique maximum at the end.

To see that $\psi_{v}$ is injective, we describe its (left-)inverse. Any walk $w$ in the image of $\psi_{v}$ has the form $b x a$, where $\max _{v}(a)=0, b$ has length $q, \bar{w}_{v}(q)>0$, and $\bar{w}_{v}(q+1)=0$. Moreover there can clearly be at most one such representation of $w$. The unique pre-image of $w$ under $\psi_{v}$ is then $a x b$.

Theorem 4.3. Let $S$ be a finite set and let $v: S \rightarrow \mathbb{R}$. Then $\left|\mathcal{M}_{v}(n)\right| \geqslant \frac{1}{2}\left|\mathcal{W}_{S}(n)\right|$.

Proof. The proof is the same as that of Theorem 2.4 we use $\psi_{v}$ in the same way to define a map $\Psi_{v}: \mathcal{W}_{S}(n)-\mathcal{M}_{v}(n) \rightarrow \mathcal{M}_{v}(n)$ and this is an injection.

Remark 4.4. Let $X \subset \mathcal{W}_{S}(n)$ be any $\Psi_{v}$-invariant subset. Then by restricting $\Psi_{v}$ to $X \cap\left(\mathcal{W}_{S}(n)-\mathcal{M}_{v}(n)\right)$, we see that $\left|X \cap \mathcal{M}_{v}(n)\right| \geqslant \frac{1}{2}|X|$. For example, we could take $X$ to be the set of $v$-closed walks, since $\Psi_{v}$ takes $v$-closed walks to $v$-closed walks. Also, note that $\Psi_{v}$ preserves the cardinality of $S$-fibers in the sense that $\left|w^{-1}(s)\right|=\left|\left(\Psi_{v} w\right)^{-1}(s)\right|$ for each $s \in S$. Hence, we could take $X$ to be the set of walks whose $S$-fibers have some prescribed cardinalities.

Remark 4.5. We can also consider weighted random walks. That is, we let $\mu$ be a probability measure on $S$, and consider the induced measure $\mu$ on $\mathcal{W}(n)$ that assigns to a walk $w$ the probability $\frac{1}{n} \sum_{s \in S}\left|w^{-1}(s)\right| \mu(s)$. Theorem 4.3 also generalizes to this case: with respect to this measure, the measure of $\mathcal{M}_{v}(n)$ is at least $\frac{1}{2}$. This works because the measure is $\Psi_{v}$-invariant, so

$$
\mu_{v}\left(\mathcal{W}_{S}(n)-\mathcal{M}_{v}(n)\right)=\sum_{w \in \mathcal{W}_{S}(n)-\mathcal{M}_{v}(n)} \mu(w)=\sum_{w \in \mathcal{W}_{S}(n)-\mathcal{M}_{v}(n)} \mu(\Psi(w)) \leqslant \sum_{w \in \mathcal{M}_{v}(n)} \mu(\Psi(w))=\mu_{v}\left(\mathcal{M}_{v}(n)\right)
$$

\section{Estimating the probability of a unique max and a unique min}

The goal of this section is to prove Theorem 5.9 which gives a $\frac{1}{4}$ asymptotic probability that a random walk with uniform step size has both a unique minimum and a unique maximum. The strategy of the proof is to show that there is a dense subset having a partition into four equal cardinality parts lying in:

$$
\mathcal{M} \cap-\mathcal{M} \quad \mathcal{M}^{c} \cap-\mathcal{M} \quad \mathcal{M} \cap(-\mathcal{M})^{c} \quad \mathcal{M}^{c} \cap(-\mathcal{M})^{c}
$$


Definition 5.1. Let $w \in \mathcal{C}(n)$. If $w \notin \mathcal{M}(n)$, we define the max-interval of $w$ to be the largest subsequence $\{p, \ldots, q\}$ of $\{1, \ldots, 2 n\}$ such that $\bar{w}(p-1)=\max (w)=\bar{w}(q)$. For $w \in \mathcal{M}(n)$, we define the max-interval of $w$ to be the max-interval of $\Psi^{-1}(w)$. Note that the max-interval subtends the part of $w$ which is modified by $\Psi$ (or $\Psi^{-1}$ ). We define the min-interval of $w$ to be the max-interval of $-w$. The size of the max-interval is its cardinality and likewise for the min-interval.

The max-interval and min-interval are generically small in the following sense:

Lemma 5.2. Let $\mathcal{U}_{+}(n, k) \subset \mathcal{C}(n)$ be the set of walks whose max-interval has size $2 k$. Similarly, $\mathcal{U}_{-}(n, k)$ denotes the walks with a size $2 k$ min-interval. For any $\epsilon>0$, there exists $N$ such that for all $n \geqslant N$ we have:

$$
\frac{1}{|\mathcal{C}(n)|}\left|\bigcup_{k=N}^{n} \mathcal{U}_{+}(n, k)\right|=\sum_{k=N}^{n} \frac{\left|\mathcal{U}_{+}(n, k)\right|}{|\mathcal{C}(n)|}<\epsilon
$$

and similarly for $\mathcal{U}_{-}$.

Proof. We will prove the claim for $\mathcal{U}_{+}$as the proof for $\mathcal{U}_{-}$is identical.

For $1 \leqslant k \leqslant n$, let $\mathcal{U}_{+}^{*}(n, k)=\mathcal{U}_{+}(n, k) \backslash \mathcal{M}(n)$. From the definitions we have $\left|\mathcal{U}_{+}^{*}(n, k)\right|=\frac{1}{2}\left|\mathcal{U}_{+}(n, k)\right|$ so it suffices to prove the claim with $\mathcal{U}_{+}$replaced by $\mathcal{U}_{+}^{*}$.

Let $\epsilon>0$. For any $1 \leqslant k \leqslant n$,

$$
\left|\mathcal{U}_{+}^{*}(n, k)\right|=|\mathcal{D}(k)| \cdot|\mathcal{M}(n-k)|=\frac{1}{k+1}\left(\begin{array}{c}
2 k \\
k
\end{array}\right) \cdot \frac{1}{2}\left(\begin{array}{c}
2(n-k) \\
n-k
\end{array}\right)
$$

Indeed, each $w \in \mathcal{U}_{+}^{*}(n, k)$ corresponds to a pair $(d, m)$ with $d \in \mathcal{D}(k)$ and $m \in \mathcal{M}(n-k)$. The correspondence arises by inserting $d$ at the maximum of $m$.

We now have the following inequality which proves the claim. Its first part holds since $\bigcup_{k=1}^{n} \mathcal{U}_{+}^{*}(n, k)=\mathcal{C}(n) \backslash \mathcal{M}(n)$ and $|\mathcal{M}(n)|=\frac{1}{2}|\mathcal{C}(n)|$ by Theorem 2.4. Its second part holds by Lemma 5.3 and its last part holds for $N$ sufficiently large by Lemma 5.4 .

$$
\sum_{k=N}^{n} \frac{\left|\mathcal{U}_{+}^{*}(n, k)\right|}{|\mathcal{C}(n)|}=\frac{1}{2}-\sum_{k=1}^{N-1} \frac{\left|\mathcal{U}_{+}^{*}(n, k)\right|}{|\mathcal{C}(n)|} \leqslant \frac{1}{2}-\sum_{k=1}^{N-1} \frac{1}{2} \frac{1}{k+1}\left(\begin{array}{c}
2 k \\
k
\end{array}\right) 4^{-k}<\epsilon
$$

Lemma 5.3. $\left(\begin{array}{c}2(n-k) \\ n-k\end{array}\right) /\left(\begin{array}{c}2 n \\ n\end{array}\right) \geqslant 4^{-k}$ for $0 \leqslant k \leqslant n$.

Proof. For each fixed $n$, we prove this by induction on $k$.

Base case:

$$
\left(\begin{array}{c}
2(n-0) \\
n-0
\end{array}\right) /\left(\begin{array}{c}
2 n \\
n
\end{array}\right)=1 \geqslant 4^{0}
$$

Inductive step: For $0 \leqslant k<n$

$$
\frac{\left(\begin{array}{c}
2(n-(k+1)) \\
n-(k+1)
\end{array}\right) /\left(\begin{array}{c}
2 n \\
n
\end{array}\right)}{\left(\begin{array}{c}
2(n-k) \\
n-k
\end{array}\right) /\left(\begin{array}{c}
2 n \\
n
\end{array}\right)}=\frac{\left(\begin{array}{c}
2(n-k)-2 \\
(n-k)-1
\end{array}\right)}{\left(\begin{array}{c}
2(n-k) \\
n-k
\end{array}\right)}=\frac{(n-k)^{2}}{(2(n-k)-1)(2(n-k))}>4^{-1}
$$


Lemma 5.4. $\sum_{k=1}^{\infty} \frac{1}{2} \frac{1}{k+1}\left(\begin{array}{c}2 k \\ k\end{array}\right) 4^{-k}=\frac{1}{2}$.

Proof. The well-known generating function for the Catalan numbers is

$$
\sum_{k=0}^{\infty} \frac{1}{k+1}\left(\begin{array}{c}
2 k \\
k
\end{array}\right) x^{k}=\frac{1-\sqrt{1-4 x}}{2 x}
$$

where this equality holds for $|x|<1 / 4$. Setting $x=1 / 4$, the left-hand side converges by the elementary estimate $\left(\begin{array}{c}2 k \\ k\end{array}\right) \leqslant \frac{4^{k}}{\sqrt{3 k+1}}$ of the central binomial coefficient. We thus obtain the following by applying $\lim _{x \nearrow \frac{1}{4}}$ to each side, and note that Abel's theorem ensures the convergence of this limit on the left.

$$
\sum_{k=0}^{\infty} \frac{1}{k+1}\left(\begin{array}{c}
2 k \\
k
\end{array}\right) 4^{-k}=2
$$

The conclusion follows since the 0 -th term of this series is 1 .

Lemma 5.5. If the max-interval and min-interval of $w$ intersect and have size $s_{1}$ and $s_{2}$, then:

$$
\max (w)-\min (w) \leqslant \frac{1}{2} \sup \left(s_{1}, s_{2}\right)
$$

Proof. Let $\{a, \ldots, b\}$ be the max-interval of $w$. By hypothesis, there is $c \in\{a, \ldots, b\}$ with $\bar{w}(c)=\min (w)$. Clearly, the difference between the maximum and minimum on a size $s$ interval is at most $s$. Since $\max (w)$ and $\min (w)$ are both attained on $\{a, \ldots, c\}$ and on $\{c, \ldots, b\}$, and since one of these intervals is of size at most $\frac{s_{1}}{2}$, it follows that $\max (w)-\min (w) \leqslant \frac{s_{1}}{2}$. Similarly, $\max (w)-\min (w) \leqslant \frac{s_{2}}{2}$.

The following is a classical fact about random walks, and we refer to [7] for an account of its history:

Lemma 5.6 (Reflection principle). For $h \geqslant 0$, the number of walks $w \in \mathcal{C}(n)$ with $\max w \geqslant h$ is equal to $\left(\begin{array}{c}2 n \\ n+h\end{array}\right)$.

Proof. For any $w \in \mathcal{C}(n)$ with $\max (w) \geqslant h$, define $R w$ by

$$
(R w)(i)= \begin{cases}w(i) ; & i \leqslant I(w) \\ -w(i) & i>I(w)\end{cases}
$$

where $I(w)$ is minimal such that $\bar{w}(i)=h$. Then $R$ is an injection onto the set of walks $w \in \mathcal{W}(2 n)$ such that $\bar{w}(2 n)=2 h$. The cardinality of the latter set is $\left(\begin{array}{c}2 n \\ n+h\end{array}\right)$.

Lemma 5.7 (Generically Disjoint). Let $\mathcal{J}(n) \subset \mathcal{C}(n)$ be the subset of walks whose maxinterval and min-interval are disjoint. Then $\lim _{n \rightarrow \infty} \frac{|\mathcal{J}(n)|}{|\mathcal{C}(n)|}=1$. 
Proof. Fix $\epsilon>0$. Let $N$ be as in Lemma 5.2 and let $n \geqslant N$. Let $\mathcal{O}(n)=\mathcal{C}(n) \backslash \mathcal{J}(n)$ consist of those walks whose max-interval and min-interval overlap. Let

$$
\mathcal{K}_{1}(n)=\mathcal{O}(n) \cap\left(\bigcup_{k=N}^{n} \mathcal{U}_{+}(n, k) \cup \bigcup_{k=N}^{n} \mathcal{U}_{-}(n, k)\right)
$$

(so that $\frac{\left|\mathcal{K}_{1}(n)\right|}{|\mathcal{C}(n)|} \leqslant 2 \epsilon$ by Lemma 5.2 and let

$$
\mathcal{K}_{2}(n)=\mathcal{O}(n) \cap\left(\bigcup_{k=1}^{N-1} \mathcal{U}_{+}(n, k) \cap \bigcup_{k=1}^{N-1} \mathcal{U}_{-}(n, k)\right)
$$

By Lemma 5.5, for any $w \in \mathcal{K}_{2}(n)$ we have $\max (w)<N$. Hence, by Lemma 5.6, we have

$$
\frac{\left|\mathcal{K}_{2}(n)\right|}{|\mathcal{C}(n)|} \leqslant \frac{\left(\begin{array}{c}
2 n \\
n
\end{array}\right)-\left(\begin{array}{c}
2 n \\
n+N
\end{array}\right)}{\left(\begin{array}{c}
2 n \\
n
\end{array}\right)}=1-\frac{(n-N+1) \cdots(n-N+N)}{(n+1) \cdots(n+N)} \stackrel{n \rightarrow \infty}{\longrightarrow} 0
$$

and so

$$
\lim _{n \rightarrow \infty} \frac{|\mathcal{O}(n)|}{|\mathcal{C}(n)|}=\lim _{n \rightarrow \infty} \frac{\left|\mathcal{K}_{1}(n)\right|}{|\mathcal{C}(n)|}+\lim _{n \rightarrow \infty} \frac{\left|\mathcal{K}_{2}(n)\right|}{|\mathcal{C}(n)|}<2 \epsilon+\lim _{n \rightarrow \infty} \frac{\left|\mathcal{K}_{2}(n)\right|}{|\mathcal{C}(n)|}=2 \epsilon
$$

Hence, $\frac{|\mathcal{J}(n)|}{|\mathcal{C}(n)|} \geqslant 1-2 \epsilon$ for every $\epsilon>0$, which proves the claim.

Lemma 5.8. $\mathcal{J}(n)$ is partitioned into 4 subsets of equal cardinality according to whether there is a unique max and/or unique min.

Proof. Since the max-interval and min-interval of elements of $\mathcal{J}(n)$ are disjoint, it is easily seen that the restrictions of the map $\Psi$ to $\mathcal{J}(n)$ leaves $\mathcal{J}(n) \cap-\mathcal{M}(n)$ invariant and hence provides bijections

$$
(\mathcal{J}(n) \cap-\mathcal{M}(n)) \backslash \mathcal{M}(n) \rightarrow(\mathcal{J}(n) \cap-\mathcal{M}(n)) \cap \mathcal{M}(n)
$$

and

$$
(\mathcal{J}(n) \backslash-\mathcal{M}(n)) \backslash \mathcal{M}(n) \rightarrow(\mathcal{J}(n) \backslash-\mathcal{M}(n)) \cap \mathcal{M}(n) .
$$

Similarly, the map $w \mapsto-\Psi(-w)$ provides bijections

$$
(\mathcal{J}(n) \backslash-\mathcal{M}(n)) \cap \mathcal{M}(n) \rightarrow(\mathcal{J}(n) \cap-\mathcal{M}(n)) \cap \mathcal{M}(n)
$$

and

$$
(\mathcal{J}(n) \backslash-\mathcal{M}(n)) \backslash \mathcal{M}(n) \rightarrow(\mathcal{J}(n) \cap-\mathcal{M}(n)) \backslash \mathcal{M}(n) .
$$

Combining these gives the desired one-to-one-to-one-to-one correspondence.

Theorem 5.9. $\lim _{n \rightarrow \infty} \frac{|\mathcal{M}(n) \cap-\mathcal{M}(n)|}{|\mathcal{C}(n)|}=\frac{1}{4}$.

Proof. Combine Lemma 5.7 and Lemma 5.8 . 
Remark 5.10. The first few terms of the sequence $|\mathcal{M}(n) \cap-\mathcal{M}(n)|$ are:

$$
0,2,4,18,64,230,852,3206,12144,46188, \ldots
$$

The convergent sequence $|\mathcal{M}(n) \cap-\mathcal{M}(n)| /|\mathcal{C}(n)|$ has the following initial terms, where $d=29099070$ :

$$
\frac{0}{d}, \frac{9699690}{d}, \frac{5819814}{d}, \frac{7482618}{d}, \frac{7390240}{d}, \frac{7243275}{d}, \frac{7223895}{d}, \frac{7248766}{d}, \frac{7268184}{d}, \frac{7274610}{d}, \ldots
$$

It is not monotonic, but we have verified that its terms are $\geqslant 1 / 4$ for all $n \geqslant 1$.

\section{Dyck words with a unique maximum}

In this section we show that, asymptotically, one half of all Dyck words have a unique maximum. We refer to [3] for a variety of other elegant counts of frequencies of various configurations within Dyck words.

We begin by describing a second, more straightforward, proof of Theorem 2.4.

Cyclic Permutation Proof. We describe a map $\mathcal{M}(n) \rightarrow \mathcal{D}(n-1)$. We cyclically permute so that the maximum appears at the beginning and end. This yields a $(2 n-1)$-to-(1) map to length $2 n$ Dyck words whose trajectories are negative except at the endpoints. After removing the first and last edges, we obtain a $(2 n-1)$-to-(1) map from $\mathcal{M}(n) \rightarrow \mathcal{D}(n-1)$. Since $|\mathcal{D}(n-1)|=\frac{1}{n}\left(\begin{array}{c}2 n-2 \\ n-1\end{array}\right)$ by Theorem 2.1, we have: $\mathcal{M}(n)=\frac{2 n-1}{n}\left(\begin{array}{c}2 n-2 \\ n-1\end{array}\right)=\frac{1}{2}\left(\begin{array}{c}2 n \\ n\end{array}\right)$.

Theorem 6.1. $\lim _{n \rightarrow \infty} \frac{|\mathcal{D}(n) \cap-\mathcal{M}(n)|}{|\mathcal{D}(n)|}=\frac{1}{2}$.

Proof. We employ the $(2 n-1)$-to- 1 map $\mathcal{M}(n) \rightarrow \mathcal{D}(n-1)$ from the above proof of Theorem 2.4. Observe that an element of $\mathcal{D}(n-1)$ has a unique minimum if and only if $(2 n-2)$ of its $(2 n-1)$ pre-images have a unique minimum.

Thus:

$$
|\mathcal{D}(n-1) \cap-\mathcal{M}(n-1)|=\frac{1}{2 n-2}|\mathcal{M}(n) \cap-\mathcal{M}(n)|
$$

and hence

$$
\lim _{n \rightarrow \infty} \frac{|\mathcal{D}(n-1) \cap \mathcal{M}(n-1)|}{|\mathcal{D}(n-1)|}=\lim _{n \rightarrow \infty} \frac{\frac{1}{2 n-2}|\mathcal{M}(n) \cap-\mathcal{M}(n)|}{\frac{1}{2 n-1}|\mathcal{M}(n)|}=\frac{1}{2}
$$

where the last equality is by Theorem 5.9 .

Remark 6.2. As in Remark 5.10, we note that $\frac{|\mathcal{D}(n) \cap-\mathcal{M}(n)|}{|\mathcal{D}(n)|} \geqslant \frac{1}{2}$ for all $n$. 


\section{References}

[1] David Blackwell and J. L. Hodges, Jr. Elementary Path Counts. Amer. Math. Monthly, 74(7):801-804, 1967.

[2] Kai Lai Chung and W. Feller. On fluctuations in coin-tossing. Proc. Nat. Acad. Sci. U. S. A., 35:605-608, 1949.

[3] Emeric Deutsch. Dyck path enumeration. Discrete Math., 204(1-3):167-202, 1999.

[4] A. Dvoretzky and Th. Motzkin. A problem of arrangements. Duke Math. J., 14:305313, 1947.

[5] Meyer Dwass. Simple random walk and rank order statistics. Ann. Math. Statist., 38:1042-1053, 1967.

[6] T. V. Narayana. Cyclic permutation of lattice paths and the Chung-Feller theorem. Skand. Aktuarietidskr, 1967:23-30, 1967.

[7] Marc Renault. Lost (and found) in translation: André's actual method and its application to the generalized ballot problem. Amer. Math. Monthly, 115(4):358$363,2008$. 